\title{
Abbau des Diglycyl-glycins und der Biuretbase im Magendarm- kanal des Hundes.
}

\author{
Von \\ Emil Abderhalden, E. S. London und Carl Voegtlin.
}

(Aus dem chemischen Institute der Universität Berlin und dem pathologischen Laboratorium des Kaiserl. Instituts für experimentelle Medizin zu St. Petersburg.)

(Der Redaktion zugegangen am 11. August 1907.)

Durch eine Reihe von Versuchen ${ }^{1}$ ) ist gezeigt worden, daß die verschiedenen Polypeptide sich gegen aktivierten Pankreassaft vom Hunde verschieden verhalten. Die einen werden gespalten, andere dagegen nicht. Nun ist von dem einen von uns in Gemeinschaft mit Y. Teruu chi ${ }^{2}$ ) nachgewiesen worden, daß Glycylglycin, das der Pankreassaft nicht in nachweisbarer Menge spaltet, von Darmsaft hydrolysiert wird. Das in diesem Falle wirksame Ferment entspricht offenbar dem Erepsin (Cohnheim), wenigstens haben wir bis jetzt keinen Anhaltspunkt für die Annahme eines besonderen Fermentes. Auch für andere Dipeptide, die nicht durch Pankreassaft angegriffen werden, ließ sich zeigen, daß sie von Darmsaft gespalten werden. Es schien uns nun von Interesse, unter natürlichen Verhältnissen festzustellen, wie die Spaltung von solchen Polypeptiden in den einzelnen Darmabschnitten verläuft, die von reinem, aus einer Pankreasfistel gewonnenem Pankreassaft nicht oder doch nur langsam hydrolysiert werden. Wir hofften auf diesem Wege einen Einblick

1) Emil Fischer und Emil Abderhalden, Über das Verhalten verschiedener Polypeptide gegen Pankreassaft und Magensaft, Diese Zeitschrift, Bd. XLVI, S. 52, 1905, und Über das Verhalten einiger Polypeptide gegen Pankreassaft, Ebenda, Bd. LI, S. 264, 1907.

2) Emil Abderhalden und Yutaka Teruuchi, Studien über die proteolytische Wirkung der Preßsäfte einiger tierischer Organe sowie des Darmsaftes, Diese Zeitschrift, Bd. XLIX, S. 1, 1906. 
in die Bedeutung und den Umfang der Erepsinverdauung in den einzelnen Darmabschnitten zu gewinnen. Die ersten Versuche dieser Art sind mit Triglycin und mit der Biuretbase (Triglycylglycinester) ausgeführt worden. Letztere wird allerdings von Pankreassaft angegriffen, jedoch sehr langsam, und der Abbau ist ein sehr unvollständiger. Die einzelnen Versuche sind in der Weise ausgeführt worden, daß Fistelhunde mit Fleisch, dem die genannten Präparate beigemischt waren, gefüttert wurden. Den Chymus fingen wir aus verschiedenen Fisteln des Magendarmkanales auf. Die Verarbeitung der einzelnen Produkte war dieselbe, wie sie schon wiederholt an dieser Stelle geschildert worden ist. Wirkönnen uns deshalb an die wesentlichsten Punkte halten. Der Chymus wurde aufgekocht, filtriert und zum Versand von St. Petersburg nach Berlin völlig eingetrocknet. Die getrocknete Masse wurde dann in Wasser gelöst, filtriert und mit so viel Wasser verdünnt, bis die Lösung etwa $1 \%$ an festen Bestandteilen aufwies. Nun fällten wir mit einem Überschuß an Phosphorwolframsäure, nutschten den Niederschlag scharf ab und preßten ihn nach wiederholtem Waschen mit Wasser gut aus. Im Filtrat der Fällung entfernten wir den Überschuß an Phosphorwolframsäure mit Baryt und dessen Überschuß dann genau mit Schwefelsäure. Das Filtrat vom Baryumsulfat wurde nun unter vermindertem Druck zur Trockene verdampft und der Rückstand in der gewohnten Weise mit Alkohol und Salzsäure verestert. Nun wurde die alkoholische Lösung der Esterchlorhydrate zunächst 12 Stunden auf Eis gestellt. War keine Abscheidung erfolgt, so wurde die Flüssigkeit eingeengt und dann versucht, durch Aufbewahren in der Kälte Krystallisation zu erhalten. Ließen sich Krystalle abscheiden, dann versuchten wir, durch Fraktionierung ein etwaiges Gemisch zu trennen. Gelang es nicht, Krystallisation einzuleiten, so wurde die Flüssigkeit völlig zur Trockene verdampft. Die Ester setzten wir dann in der bekannten Weise aus der alkoholischen Lösung des Rückstandes mit der auf den Chlorgehalt berechneten Menge Natriumalkoholat in Freiheit. Sie wurden dann destilliert. Das Destillat verdampften wir nach Zusatz von wässeriger Salzsäure zur Trockene und veresterten dann den Rückstand noch einmal. 
Den Phosphorwolframsäurerückstand zerlegten wir in der gewohnten Weise mit Baryt, entfernten aus dem Filtrat vom phosphorwolframsauren Baryt quantitativ den Baryt und verdampften das Filtrat vom Baryumsulfat unter vermindertem Druck zur Trockene. Den Rückstand veresterten wir mit Alkohol und Salzsäure. Bei den Versuchen mit Diglycyl-glycin haben wir die Zerlegung des Phosphorwolframsäureniederschlages nur zur Kontrolle vorgenommen. In der angewandten Verdünnung fällt Diglycyl-glycin mit Phosphorwolframsäure nicht.

Es sind folgende Versuche ausgeführt worden:

I. Versuche mit Diglycyl-glycin.

a) Versuch am Pylorusfistelhund Usaty. Er erhielt $200 \mathrm{~g}$ Fleisch $+1,25 \mathrm{~g}$ Diglycyl-glycin. Während des Versuches wurden $160 \mathrm{ccm}$ peptischer Verdauungsprodukte in das Duodenum eingeführt. Nach den einzelnen Injektionen sistierte die Ausscheidung von Chymus 3-5 Minuten. An Chymus wurden $512 \mathrm{~g}$ erhalten. Isoliert wurden 1,0 g Diglycyl-glycinesterchlorhydrat vom Zersetzungspunkt $214-220^{\circ}$ (korr.). Diese Menge entspricht $0,75 \mathrm{~g}$ Diglycyl-glycin. Aus der Mutterlauge konnten wir weder Glycyl-glycin noch Glykokoll gewinnen. Eine Spaltung des Diglycyl-glycins war somit offenbar nicht erfolgt.

b) Versuch am Pylorusfistelhund ohne Einschaltung des Duodenalreflexes. Das Versuchstier erhielt $200 \mathrm{~g}$ Fleisch + $4,0 \mathrm{~g}$ Diglycyl-glycin. Der Chymus wurde in Intervallen von 5 bis 45 Sekunden ausgeschieden. Die Entleerung dauert 1 Stunde und 30 Minuten. Der ganze Brei wog 406 g. Erhalten wurden $3,5 \mathrm{~g}$ Diglycyl-glycinesterchlorhydrat vom Zersetzungspunkt $215-220^{\circ}$ (korr.) und einen Chlorgehalt von 13,78\%. (Berechnet für $\mathrm{C}_{8} \mathrm{H}_{16} \mathrm{~N}_{3} \mathrm{O}_{4} \cdot \mathrm{Cl}=13,98 \% \mathrm{Cl}$.) Auch hier konnten wir kein Glycyl-glycin und kein Glykokoll isolieren. Ersteres suchten wir als Anhydrid zu gewinnen und letzteres als Esterchlorhydrat.

c) Duodenalfistelhund: Verfütterung von $200 \mathrm{~g}$ Fleisch + 1,42 g Diglycyl-glycin. Stelle der Fistel: Ende des Duodenums. Dauer des Versuches 5 Stunden. Das Gesamtgewicht des Chymus betrug $735 \mathrm{~g}$. Isoliert 0,55 g Glykokollesterchlor- 
hydrat. Weder Diglycyl-glycin noch Glycyl-glycin waren nachweisbar. Da bis jetzt bei allen Untersuchungen Glykokoll im Chymus des Duodenums bei Fleischfütterung nicht aufgefunden worden ist, darf wohl angenommen werden, daß das isolierte Glykokoll auf das verfütterte Diglycyl-glycin zurückzuführen ist.

d) Stelle der Fistel: $1 \mathrm{~m}$ vor dem Coecum. Verfüttert 200 g Fleisch $+1,4$ g Diglycyl-glycin. Dauer des Versuches 9 Stunden. Gewicht des aufgefangenen Chymus $130 \mathrm{~g}$. Es ließ sich weder Diglycyl-glycin noch ein Abbauprodukt dieses Tripeptids nachweisen. Offenbar war schon alles zur Resorption gelangt.

e) Fistel $2-3 \mathrm{~cm}$ vor dem Coecum. Verfüttert $500 \mathrm{~g}$ Fleisch $+0,9 \mathrm{~g}$ Diglycyl-glycin. Dauer des Versuches $13^{1 / 2}$ Stunden. Gewicht des Chymus $130 \mathrm{~g}$. Auch hier fahndeten wir vergeblich auf Diglycyl-glycin und auf Abbauprodukte. Aus diesen Versuchen geht hervor, daß das Diglycyl-glycin, das durch Pankreassaft nicht oder doch nur in geringem Umfange angegriffen wird, bereits im Duodenum energisch zur Spaltung kommt.

II. Versuche mit Triglycyl-glycinäthylester (Biuretbase von Curtius).

a) Pylorusfistelhund Usaty: Fütterung mit $700 \mathrm{~g}$ Fleisch $+2,4 \mathrm{~g}$ Biuretbase. Während des $5^{1 / 6}$ Stunden dauernden Versuches wurden in das Duodenum Produkte peptischer Verdauung eingeführt. Nach den Injektionen blieb der Pylorus jeweilen 7-10 Minuten geschlossen. Das Gewicht des gesamten Chymus betrug $707 \mathrm{~g}$. Auch hier wurde zunächst aus sehr verdünnter ca. 1\% iger Lösung mit Phosphorwolframsäure gefällt und Niederschlag und Filtrat für sich bearbeitet. W'ir suchten in beiden Portionen getrennt mit Hilfe der Estermethode nach schwer löslichen Esterchlorhydraten. Aus dem zerlegten Phosphorwolframsäureniederschlag konnten wir weder Triglycylglycinesterchlorhydrat noch Abbauprodukte der Biuretbase isolieren, dagegen gelang es, aus dem nicht mit Phosphorwolframsäure fällbaren Teil einen schwer löslichen salzsauren Ester abzuscheiden. Er schmolz gegen $210^{\circ}$ und zeigte einen Chlorgehalt von 11,95\%. Berechnet sind für den salzsauren Triglycylglycinäthylester $\mathrm{C}_{10} \mathrm{H}_{19} \mathrm{O}_{5} \mathrm{~N}_{4} \mathrm{Cl} 11,41 \% \mathrm{Cl}$. Es lag somit offenbar 
unverändertes Tetrapeptid vor. Die Menge des reinen Produktes betrug allerdings nur $0,75 \mathrm{~g}$. Aus der Mutterlauge isolierten wir eine weniger reine Substanz, die zwischen 190 und $200^{\circ}$ schmolz und einen Chlorgehalt von 12,25\% zeigte. Ihre Menge betrug $0,81 \mathrm{~g}$. Aus der Mutterlauge dieser zweiten Fraktion konnten wir keine krystallinische Abscheidung mehr erhalten. Wir dampften sie deshalb völlig zur Trockene ein und setzten die Ester aus der alkoholischen Lösung der Esterchlorhydrate mit der auf den Chlorgehalt berechneten Menge Natriumalkoholat in Freiheit und destillierten die filtrierte Flüssigkeit. Das Destillat hinterließ beim Eindampfen mit wässeriger Salzsäure einen Rückstand, den wir mit Alkohol und Salzsäure veresterten. Bald schieden sich Kryställchen ab. Ihre Menge betrug 0,25 g. Sie schmolzen gegen $144^{\circ}$. Es lag somil Glykokollesterchlorhydrat vor. Es ist fraglich, ob wir dieses Glykokoll auf eine Fermenthydrolyse zurückzuführen haben, oder ob nicht vielmehr Glykokoll bei der Veresterung in geringen Mengen abgespalten worden ist. Es muß auch in Erwägung gezogen werden, ob die verwendete Biuretbase ganz rein war. Sie ist zwar mit aller Sorgfalt dargestellt worden und zwar aus Glykokollester, wir können jedoch trotzdem nicht für völlige Reinheit garantieren. Unser Versuch ist vorläufig als nicht ganz eindeutig anzusehen.

b) Fistel am Ende des Duodenums. Verfütterung von $200 \mathrm{~g}$ Fleisch + 2,66 g Biuretbase. Dauer des Versuches $4^{\mathbf{1} / \mathbf{3}}$ Stunden. Gewicht des aufgesammelten Chymus 695 g. Unverändertes Ausgangsprodukt konnten wir nicht isolieren, dagegen $1,5 \mathrm{~g}$ Glykokollesterchlorhydrat und $0,35 \mathrm{~g}$ Glycinanhydrid ( $\mathrm{F}$. $=240^{\circ}$ ). Offenbar war die verabreichte Biuretbase gespalten worden, denn bisher haben wir im Chymus des Duodenums Glykokoll nicht aufgefunden.

c) Fistel $1 \mathrm{~m}$ vom Pylorus entfent. $300 \mathrm{~g}$ Fleisch + $2,10 \mathrm{~g}$ Biuretbase verfüttert. Dauer des Versuches $6^{1 / 2}$ Stunden. Aufgefangener Brei $384 \mathrm{~g}$. Wir konnten hier nur Glykokoll als Esterchlorhydrat nach erfolgter Infreiheitsetzung der Ester nachweisen und zwar gewannen wir $0,68 \mathrm{~g}\left(\mathrm{~F} .=144^{\circ}\right)$. Glycinanhydrid ließ sich im Destillationsrückstand nicht zur Abscheidung bringen. 
d) Fistel $1 \mathrm{~m}$ vor dem Coecum. $300 \mathrm{~g}$ Fleisch $+3,50 \mathrm{~g}$ Biuretbase verfüttert. Dauer des Versuches $8^{1 / 2}$ Stunden. Der gesammelte Chymus wog $287 \mathrm{~g}$. Es gelang uns nicht, irgend ein Abbauprodukt der Biuretbase nachzuweisen. Offenbar waren sie alle schon zur Resorption gelangt.

e) Fistel $2-3 \mathrm{~cm}$ vor dem Coecum. Verfütterung von $200 \mathrm{~g}$ Fleisch + 2,34 g Biuretbase. Dauer des Versuches 12 Stunden. Der gesamte Chymus wog $39 \mathrm{~g}$. Auch hier bemühten wir uns vergeblich, Glykokoll oder ein höheres Abbauprodukt der Biuretbase $\mathrm{zu}$ isolieren.

Aus diesen Untersuchungen geht hervor, daß aller Wahrscheinlichkeit nach Diglycyl-glycin und Triglycyl-glycinäthylester im Magen nicht oder doch nur in geringem Umfange angegriffen werden und lange Zeit nachweisbar sind, während im Darm der Abbau rasch einsetzt und mit ihm offenbar auch die Resorption. 